\title{
Exposing the Causal Effect of Body Mass Index on the Risk of Type 2 Diabetes Mellitus: A Mendelian Randomization Study
}

\author{
Liang Cheng ${ }^{1}$, He Zhuang ${ }^{1}$, Hong Jü ${ }^{2}$, Shuo Yang ${ }^{1}$, Junwei Han ${ }^{1 *}$, Renjie Tan ${ }^{1 *}$ and \\ Yang $\mathrm{Hu}^{3 *}$ \\ ${ }^{1}$ College of Bioinformatics Science and Technology, Harbin Medical University, Harbin, China, ${ }^{2}$ Department of Information \\ Engineering, Heilongjiang Biological Science and Technology Career Academy, Harbin, China, ${ }^{3}$ School of Life Sciences \\ and Technology, Harbin Institute of Technology, Harbin, China
}

\section{OPEN ACCESS}

Edited by:

Tao Huang,

Shanghai Institutes for Biological Sciences (CAS), China

Reviewed by:

Chong Wang,

Brigham and Women's Hospital and Harvard Medical School,

United States

Sicheng Hao,

Northeastern University, United States

Leyi Wei,

Tianjin University, China

*Correspondence:

Junwei Han

hanjunwei1981@163.com

Reniie Tan

renjie.tan@outlook.com Yang $\mathrm{Hu}$

huyang@hit.edu.cn

Specialty section:

This article was submitted to Bioinformatics and Computational

Biology,

a section of the journal

Frontiers in Genetics

Received: 21 October 2018 Accepted: 29 January 2019

Published: 14 February 2019

Citation:

Cheng L, Zhuang $H$, Ju H, Yang S, Han J, Tan R and Hu Y (2019) Exposing the Causal Effect of Body Mass Index on the Risk of Type 2 Diabetes Mellitus: A Mendelian

Randomization Study

Front. Genet. 10:94.

doi: 10.3389/fgene.2019.00094
Introduction: High body mass index (BMI) is a positive associated phenotype of type 2 diabetes mellitus (T2DM). Abundant studies have observed this from a clinical perspective. Since the rapid increase in a large number of genetic variants from the genome-wide association studies (GWAS), common SNPs of BMI and T2DM were identified as the genetic basis for understanding their associations. Currently, their causality is beginning to blur.

Materials and Methods: To classify it, a Mendelian randomisation (MR), using genetic instrumental variables (IVs) to explore the causality of intermediate phenotype and disease, was utilized here to test the effect of BMI on the risk of T2DM. In this article, MR was carried out on GWAS data using 52 independent BMI SNPs as IVs. The pooled odds ratio $(\mathrm{OR})$ of these SNPs was calculated using inverse-variance weighted method for the assessment of $5 \mathrm{~kg} / \mathrm{m}^{2}$ higher BMI on the risk of T2DM. The leave-one-out validation was conducted to identify the effect of individual SNPs. MR-Egger regression was utilized to detect potential pleiotropic bias of variants.

Results: We obtained the high OR $(1.470 ; 95 \% \mathrm{Cl} 1.170$ to $1.847 ; P=0.001)$, low intercept $(0.004, P=0.661)$, and small fluctuation of ORs ffrom -0.039 [(1.412 - 1.470) / 1.470)] to 0.075 [(1.568-1.470) / 1.470)] in leave-one-out validation.

Conclusion: We validate the causal effect of high BMI on the risk of T2DM. The low intercept shows no pleiotropic bias of IVs. The small alterations of ORs activated by removing individual SNPs showed no single SNP drives our estimate.

Keywords: body mass index, type 2 diabetes mellitus, casual effect, Mendelian randomisation, phenotype

\section{INTRODUCTION}

Diabetes mellitus (DM) is characterized by a bunch of chronic metabolic diseases leading to insulinsecretion deficiency (Olokoba et al., 2012; Pan et al., 2013; Shi and Hu, 2014). High blood sugar levels in DM patients over a prolonged period impair body tissues, such as eye, kidney, heart, and so on. Currently, more than 400 million people suffer from diabetes worldwide, of which type 2 DM (T2DM) makes up about 90\% (Olokoba et al., 2012; Pan et al., 2013; Shi and Hu, 2014). Most patients who suffer from T2DM are over the age of 40 (Olokoba et al., 2012; Pan et al., 2013; 
Shi and $\mathrm{Hu}, 2014)$. In theory, people have a long time to prevent T2DM under the right direction. To this end, researchers go out of their way to investigate the causes of T2DM.

Observational studies exposed that body mass index (BMI) was strongly associated with the risk of being diagnosed with T2DM (Sanada et al., 2012; Ganz et al., 2014; Chen et al., 2015, 2016; Zhao et al., 2017). In Sanada et al. (2012) conducted a 10-year retrospective cohort study on 969 men and 585 women (Sanada et al., 2012). They observed high BMI was an independent and dose-dependent risk factor for T2DM in Japanese patients (Sanada et al., 2012). In Ganz et al. (2014) directed a case-control study to assess the association between BMI and the risk of T2DM in the United States (Ganz et al., 2014). A positive association between them was found in 12,179 cases ( $>=18$ years old) and 25,177 controls (Ganz et al., 2014). The analogous studies without considering genetic factors almost came to a consistent conclusion.

After identifying a large number of BMI-associated and T2DM-associated loci in genome-wide association studies (GWAS), their common associated variants were then interpreted as the underlying cause of BMI and the risk of T2DM. In 2007, the first common variant in the FTO gene of BMI and T2DM was reported in European descents (Frayling et al., 2007). Subsequently, corresponding investigations sprung up for validating the existing common locus and identifying their novel common variants of BMI and T2DM (Andreasen et al., 2008; Herder et al., 2008; Cauchi et al., 2009; Legry et al., 2009; Webster et al., 2010; Song et al., 2012; Xi et al., 2014). In 2014, a meta-analysis of 42 studies for BMI and T2DM associated variants was conducted (Xi et al., 2014). Eventually, 4 statistically significant associated variants (FTO rs9939609, SH2B1 rs7498665, FAIM2 rs7138803, GNPDA2 rs10938397) were identified for both in Europeans.

Whether a higher BMI increases the risk of T2DM or T2DM affects BMI or their common genetic factors take effect, is still unknown according to current observations. In addition, after considering confounding factors, the causal relationship between BMI and T2DM may be reverse. To estimate the causal effect of BMI on the risk of T2DM, we conducted this Mendelian randomization (MR) study, which is an instrumental variable (IV) based method to infer causality of exposure and disease in observation studies. Genetic variants that are associated with intermediate phenotypic exposures are introduced as IVs by $\mathrm{MR}$ to estimate the effect of phenotypic exposures on a disease outcome (Figure 1A). Due to random distribution of gene variants during gametogenesis, IV-based analysis can avoid reverse causality. The basic principle of estimating the influence of BMI on the risk of T2DM using MR is shown in the Figure 1B, where $\mathrm{Z}$ (e.g., variants) represents IV, X indicates exposure BMI, and $\mathrm{Y}$ is disease T2DM. Two assumptions should be suitable for the case before using MR.

(1) The variants are robustly associated with BMI.

(2) The variants are independent of the T2DM without considering BMI and confounders. It means the only way to influence the T2DM by the variants is via an intermediate.
The two assumptions mean the variants should be associated with BMI but not with T2DM. Therefore, the conclusions based on MR could not result from the common genetic factors of BMI and T2DM.

\section{MATERIALS AND METHODS}

Two summary-level data of GWAS datasets were utilized by MR analysis. One of them was for extracting significant BMI SNP sets to meet the assumption 1 . And the other was for extracting no significant T2DM SNP sets to meet assumption 2. The intersections of these two SNP sets were then analyzed using MR.

\section{Summary-Level Data for Associations Between Genetic Variants and BMI}

In Locke et al. (2015) conducted a meta-analysis of BMI using GWAS on Metabochip studies (Voight et al., 2012). Totally, 322,154 individuals of European descents and 17,072 individuals of non-European descent were analyzed. As a result, 97 BMIassociated SNPs $\left(P<5 \times 10^{-8}\right)$ were identified for European. The corresponding SNPs, effect allele (EA), allele frequencies, beta coefficients, and standard errors (SEs) were extracted from Genetic Investigation of Anthropometric Traits (GIANT) consortium (Locke et al., 2015) as summary-level data for associations between genetic variants and BMI.

\section{Summary-Level Data for Associations Between Genetic Variants and T2DM}

Morris et al. (2012) carried out a combined meta-analysis of European descents on two GWAS data sets (Yang et al., 2010; Lee et al., 2011), which involved 22,669 cases and 58,119 controls. All the variants were then genotyped with Metabochip involving 1,178 cases and 2,472 controls of Pakistani descent. The analytical result contains novel susceptibility locus together with other SNPs, SEs and their P-values on the risk of T2DM. These were utilized as summary-level data for associations between genetic variants and T2DM.

\section{Data Processing and Analysis}

Two summary-level datasets were processed into assumptionoriented data (Figure 2). According to assumption 2, genetic pleiotropy can result in over-precise estimates in subsequent analysis. According to the application principles of Mendelian randomization analysis, the study is based on Mendel's second law of inheritance: the separation and combination of genetic gametes controlling different traits do not interfere with each other; in the formation of gametes, the paired genetic gametes that determine the same trait are separated from each other, and the genetic gametes that determine different traits are freely combined. When the two genes are not completely independent, they will show a certain degree of linkage, a situation called linkage disequilibrium (LD), which will greatly affect the exclusiveness of the variable tool to phenotypic inheritance, leading the subsequent calculations bias generally called "over-precise estimates." To avoid this situation, these 
A

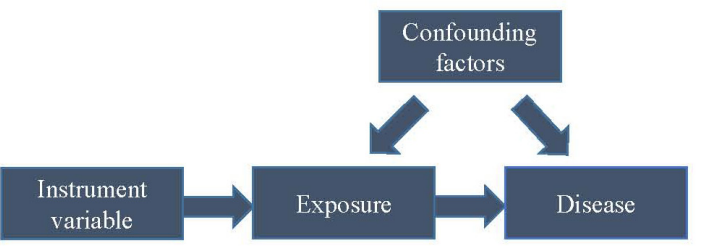

B

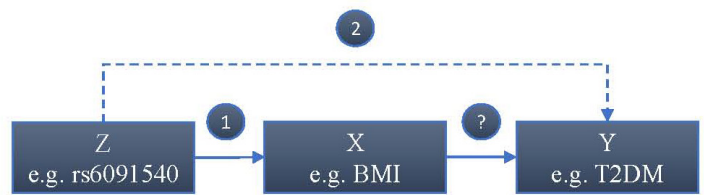

FIGURE 1 | Mendelian randomisation analysis using genetic variants as instrumental variables for estimating the influence of BMI on T2DM. (A) Causal effect in Mendelian randomisation. (B) The basic principle of estimating the influence of BMI on the risk of T2DM.

\section{Summary-level data}

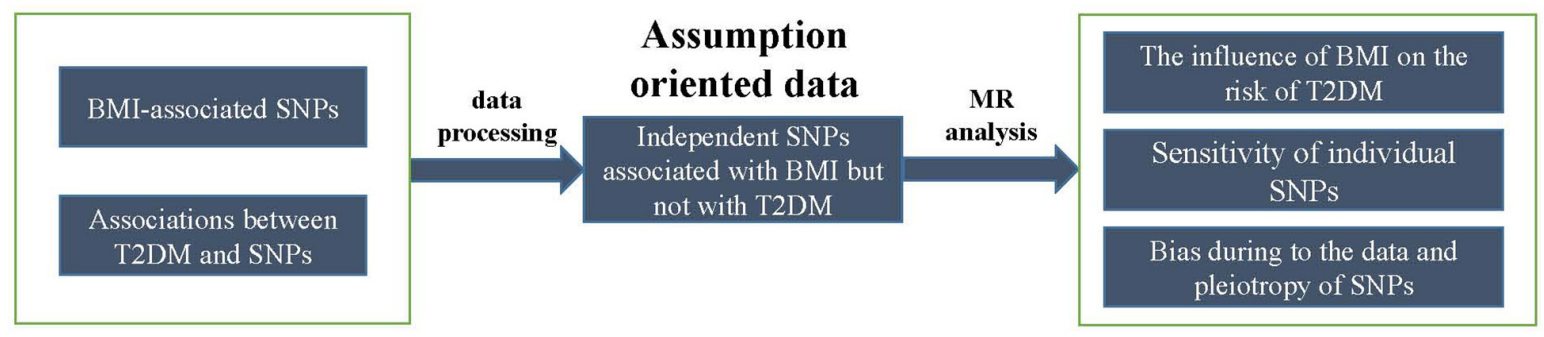

FIGURE 2 | The schematic of data processing and analysis.

loci with potential LD were removed from 97 BMI-associated SNPs, which was done by Noyce et al. (2017) in the previous study. The 97 SNPs were first ranked from the smallest to largest $P$-values. Then for the top ranked SNPs, Noyce et al. (2017) removed those in LD $\left(R^{2}\right.$ threshold of 0.001$)$ or those within $10,000 \mathrm{~kb}$ physical distance based on a reference dataset (Devuyst, 2015) from the 97 SNPs. This process was iterated for the remaining SNPs. As a result, 78 BMI-associated SNPs $\left(P<5 \times 10^{-8}\right)$ without potential LD of each other were obtained. According Xi et al. (2014), meta-analysis, four SNPs (rs9939609, rs7498665, rs7138803, rs10938397) were found at the T2DM-associated locus, and were also further removed from these 78 SNPs. In addition, those SNPs with $P$-value less than 0.05 by Morris et al. (2012) were removed as well. Finally, 52 SNPs that confirmed to the two MR assumptions were retained for MR analysis.

Three subjects involving the influence of BMI on the risk of T2DM (Figure 2), the sensitivity of the disproportionate effects of variants, and the detection of bias due to pleiotropy were investigated in MR analysis. These issues were analyzed by MR method, leave-one-out validation, and MR-Egger regression (Bowden et al., 2015), respectively.

\section{- MR method}

MR method was described in the previous study (Bowden et al., 2015) and summarized for evaluating the influence of BMI on the risk of T2DM as below. Assuming $X, Y$, and $Z$ are BMI, T2DM, and variants, respectively, Wald ratio $\left(\beta_{X Y}\right)$ of BMI to T2DM through specified variant is calculated as follows:

$$
\beta_{X Y}=\beta_{Z Y} / \beta_{Z X}
$$

where $\beta_{Z Y}$ represents the per-allele $\log (O R)$ of T2DM from summary-level data of Morris et al. (2012) study. $\beta_{Z X}$ is the perallele $\log (\mathrm{OR})$ of BMI from summary-level data of Locke et al. (2015) study. SE of BMI-T2DM association of each Wald ratio is defined as follows:

$$
S E_{X Y}=S E_{Z Y} / S E_{Z X}
$$

where $S E_{Z Y}$ and $S E_{Z X}$ represent the $S E$ of the variant-T2DM and variant-BMI associations from corresponding summarylevel data, respectively. Subsequently, 95\% confidence intervals (CIs) were then calculated from the $S E$ of each Wald ratio. These summarized data were then estimated using inversevariance weighted (IVW) linear regression for meta-analysis. The meta-analysis model for the point estimate is according to the heterogeneity of the summarized data. Fixed effect model is used for no significant heterogeneity, and random-effect model is used for others.

To evaluate the genetic heterogeneity of summarized data, Cochran's $Q$-test and statistic $I^{2}$ were utilized here. Cochran's $Q$-test follows a $\chi^{2}$ distribution with $k-1$ degrees of freedom, where $\mathrm{k}$ represents the number of variants for analysis. $I^{2}=(Q-(k-1)) / Q \times 100 \%$ ranges from 0 to $100 \% . P<0.01$ and $I^{2}>50 \%$ were defined as the significant heterogeneity here (Zhang et al., 2015).

\section{- Leave-one-out validation}

To test the sensitivity of variants, we designed a leave-one-out validation measure. In brief, to test the influence of an SNP to the conclusion, the SNP was removed from the 52 SNPs to carry out IVW point estimate. The fluctuation of the results before and after removing the SNP reflects the sensitivity of this SNP. Here this process was iterated for each of these 52 SNPs. 
TABLE 1 | Associations of genetic variants with BMl and T2DM.

\begin{tabular}{|c|c|c|c|c|c|c|c|c|c|}
\hline SNP & Chr & Gene & BP & $\begin{array}{l}\text { BMI } \\
\text { beta }\end{array}$ & BMI SE & BMI P & $\begin{array}{l}\text { T2DM } \\
\text { beta }\end{array}$ & $\begin{array}{c}\text { T2DM } \\
\text { SE }\end{array}$ & $\begin{array}{c}\text { T2DM } \\
\mathbf{P}\end{array}$ \\
\hline rs977747 & 1 & $\operatorname{TAL} 1(\mathrm{~N}) \operatorname{TAL} 1(\mathrm{~N})$ & 47457264 & 0.017 & 0.003 & $2.18 \mathrm{E}-08$ & -0.010 & 0.020 & 0.63 \\
\hline rs3101336 & 1 & NEGR1(B,C,D,N) & 72523773 & 0.032 & 0.003 & 2.66E-26 & 0.010 & 0.020 & 0.66 \\
\hline rs12401738 & 1 & FUBP1(N); USP33(D) & 78219349 & 0.02 & 0.003 & $1.15 \mathrm{E}-10$ & 0.000 & 0.020 & 0.86 \\
\hline rs11165643 & 1 & PTBP2(D,N) & 96696685 & 0.022 & 0.003 & $2.07 \mathrm{E}-12$ & 0.030 & 0.019 & 0.11 \\
\hline rs2820292 & 1 & NAV1(N) & 200050910 & 0.018 & 0.003 & $1.83 \mathrm{E}-10$ & -0.010 & 0.020 & 0.45 \\
\hline rs10182181 & 2 & $\operatorname{NCOA1(B)}$ & 25003800 & 0.031 & 0.003 & $8.78 \mathrm{E}-24$ & -0.020 & 0.015 & 0.34 \\
\hline rs1016287 & 2 & LINC01122(N) & $59,159,129$ & 0.0229 & 0.0034 & $2.25 \mathrm{E}-11$ & 0.030 & 0.019 & 0.17 \\
\hline rs2121279 & 2 & LRP1B(N) & 142759755 & 0.024 & 0.004 & $2.31 \mathrm{E}-08$ & 0.030 & 0.024 & 0.18 \\
\hline rs1460676 & 2 & FIGN(N) & 164275935 & 0.021 & 0.004 & 4.98E-08 & 0.020 & 0.024 & 0.49 \\
\hline rs1528435 & 15 & UBE2E3(N) & 65864222 & 0.018 & 0.003 & $1.20 \mathrm{E}-08$ & 0.020 & 0.020 & 0.21 \\
\hline rs6804842 & 3 & $\operatorname{RARB}(\mathrm{B})$ & 25081441 & 0.018 & 0.003 & $2.48 \mathrm{E}-09$ & 0.020 & 0.020 & 0.21 \\
\hline rs2365389 & 3 & $\mathrm{FHIT}(\mathrm{N}$ & 61211502 & 0.02 & 0.003 & $1.63 \mathrm{E}-10$ & -0.010 & 0.015 & 0.7 \\
\hline rs13078960 & 3 & $\mathrm{CADM} 2(\mathrm{D}, \mathrm{N})$ & 85890280 & 0.029 & 0.004 & $1.74 \mathrm{E}-14$ & 0.020 & 0.020 & 0.44 \\
\hline rs16851483 & 3 & RASA2(N) & 142758126 & 0.048 & 0.008 & $3.55 \mathrm{E}-10$ & -0.010 & 0.034 & 0.82 \\
\hline rs13107325 & 4 & SLC39A8(M,N,Q) & 103407732 & 0.047 & 0.007 & $1.83 \mathrm{E}-12$ & 0.039 & 0.042 & 0.38 \\
\hline rs11727676 & 4 & $\mathrm{HHIP}(\mathrm{B}, \mathrm{N})$ & 145878514 & 0.037 & 0.006 & $2.55 \mathrm{E}-08$ & -0.077 & 0.045 & 0.12 \\
\hline rs205262 & 6 & C6orf106(N); SNRPC(Q) & 34671142 & 0.021 & 0.003 & $1.75 \mathrm{E}-10$ & 0.000 & 0.020 & 0.97 \\
\hline rs2033529 & 6 & TDRG1(N); LRFN2(D) & 40456631 & 0.018 & 0.003 & $1.39 \mathrm{E}-08$ & 0.020 & 0.020 & 0.32 \\
\hline rs2207139 & 6 & TFAP2B(B,N) & 50953449 & 0.045 & 0.004 & 4.13E-29 & 0.039 & 0.024 & 0.14 \\
\hline rs9400239 & 6 & FOXO3(B,N); HSS00296402(Q) & 109084356 & 0.017 & 0.003 & $1.61 \mathrm{E}-08$ & 0.010 & 0.020 & 0.62 \\
\hline rs10968576 & 9 & LINGO2(D,N) & 28404339 & 0.025 & 0.003 & $6.61 \mathrm{E}-14$ & 0.000 & 0.020 & 1 \\
\hline rs6477694 & 9 & EPB41L4B(N); C9orf4(D) & 110972163 & 0.017 & 0.003 & $2.67 \mathrm{E}-08$ & 0.010 & 0.020 & 0.42 \\
\hline rs1928295 & 9 & TLR4(B,N) & 119418304 & 0.018 & 0.003 & 7.91E-10 & 0.030 & 0.015 & 0.12 \\
\hline rs10733682 & 9 & $\operatorname{LMX} 1 \mathrm{~B}(\mathrm{~B}, \mathrm{~N})$ & 128500735 & 0.019 & 0.003 & $1.83 \mathrm{E}-08$ & 0.030 & 0.019 & 0.057 \\
\hline rs7899106 & 10 & GRID1(B,N) & 87400884 & 0.038 & 0.007 & $2.96 \mathrm{E}-08$ & -0.020 & 0.034 & 0.67 \\
\hline rs11030104 & 11 & $\operatorname{BDAF}(\mathrm{B}, \mathrm{M}, \mathrm{N})$ & 27641093 & 0.042 & 0.004 & $5.56 \mathrm{E}-28$ & 0.010 & 0.025 & 0.49 \\
\hline rs12286929 & 11 & CADM1(N) & 114527614 & 0.021 & 0.003 & $1.31 \mathrm{E}-12$ & 0.010 & 0.020 & 0.5 \\
\hline rs11057405 & 12 & $\mathrm{CLIP} 1(\mathrm{~N})$ & 121347850 & 0.03 & 0.005 & $2.02 \mathrm{E}-08$ & -0.095 & 0.044 & 0.055 \\
\hline rs10132280 & 14 & STXBP6(N) & 24998019 & 0.022 & 0.003 & $1.14 \mathrm{E}-11$ & 0.030 & 0.019 & 0.12 \\
\hline rs3736485 & 15 & SCG3(B,D); DMXL2(M,N) & 49535902 & 0.016 & 0.003 & 7.41E-09 & 0.020 & 0.015 & 0.29 \\
\hline rs16951275 & 2 & M4P2K5(B,D,N); LBXCOR1(M) & 181259207 & 0.03 & 0.004 & $1.91 \mathrm{E}-17$ & 0.030 & 0.019 & 0.21 \\
\hline rs758747 & 16 & $\mathrm{NLRC3(N)}$ & 3567359 & 0.023 & 0.004 & 7.47E-10 & 0.000 & 0.025 & 0.97 \\
\hline rs3888190 & 16 & ATXN2L(Q); SBK1(Q,D); SULT1A2(Q); TUFM(Q) & 28796987 & 0.031 & 0.003 & $3.14 \mathrm{E}-23$ & 0.010 & 0.015 & 0.77 \\
\hline rs1000940 & 17 & RABEP1(N) & 5223976 & 0.018 & 0.003 & $1.28 \mathrm{E}-08$ & 0.010 & 0.025 & 0.49 \\
\hline rs1808579 & 18 & NPC1(B,G,M,Q); C18orf8(N,Q) & 19358886 & 0.016 & 0.003 & 4.17E-08 & 0.030 & 0.019 & 0.13 \\
\hline rs7239883 & 18 & LOC284260(N); RIT2(B,D) & 38401669 & 0.015 & 0.003 & $1.51 \mathrm{E}-08$ & 0.020 & 0.015 & 0.34 \\
\hline rs29941 & 14 & KCTD15(N) & 78969207 & 0.018 & 0.003 & $2.41 \mathrm{E}-08$ & 0.000 & 0.020 & 0.92 \\
\hline rs2287019 & 19 & QPCTL(N); GIPR(B,M) & 50894012 & 0.035 & 0.004 & $4.59 \mathrm{E}-18$ & -0.030 & 0.029 & 0.33 \\
\hline rs6091540 & 20 & ZFP64(N) & 50521269 & 0.019 & 0.003 & $2.15 \mathrm{E}-11$ & 0.010 & 0.020 & 0.8 \\
\hline rs2836754 & 21 & ETS2(N) & 39213610 & 0.017 & 0.003 & 1.61E-08 & -0.020 & 0.020 & 0.18 \\
\hline
\end{tabular}




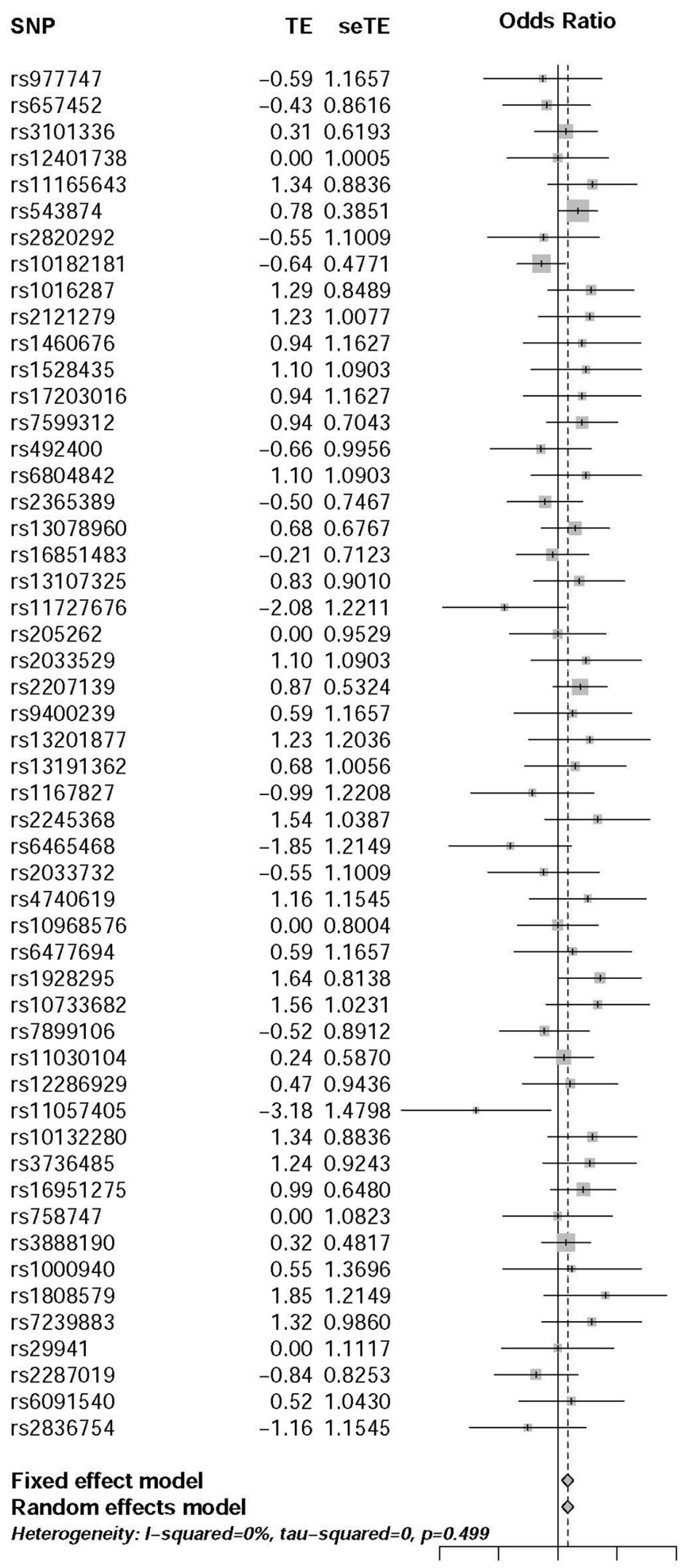

\section{SNP}

rs977747

rs3101336

rs12401738

rs11165643

rs212127

rs1460676

rs1528435

S17203016

rs6804842

rs2365389

rs13078960

rs16851483

rs13107325

rs205262

rs2033529

rs2207139

rs1167827

rs2245368

rs6465468

rs1928295

rs10733682

rs7899106

s11030104

rs16951275

rs758747

rs3888190

rs2287019

rs6091540

Fixed effect model

Random effects mode

Heterogeneity: $I$-squared $=0 \%$, tau-squared $=0, p=0.499$
$-0.591 .1657$

$-0.430 .8616$

0.310 .6193

1.340 .8836

0.780 .3851

$-0.551 .1009$

1.231 .0077

0.941 .1627

1.101 .0903

0.940 .7043

$-0.660 .9956$

1.101 .0903

$-0.210 .7123$

0.830 .9010

$-2.081 .2211$

$0.00 \quad 0.9529$

1.101 .0903

0.870 .5324

0.591 .1657

1.231 .2036

$-0.991 .2208$

1.541 .0387

$-1.851 .2149$

$-0.551 .1009$

1.640 .8138

1.561 .0231

$\begin{array}{lll}0.52 & 0.8912 \\ 0.24 & 0.5870 \\ 0.47 & 0.9436\end{array}$

1.340 .8836

1.240 .9243

0.990 .6480

0.001 .0823

0.551 .3696

1.851 .2149

1.320 .9860

0.001 .1117

$-0.840 .8253$

0.521 .0430

$-1.161 .1545$
$-0.520 .8912$

\section{OR $\quad 95 \%-C l$ W(fixed) $W($ random)}

$0.56 \quad[0.06 ; 5.47]$

$0.65[0.12 ; 3.51]$

$1.36 \quad[0.41 ; 4.59]$

$1.00[0.14 ; 7.11]$

$3.83[0.68 ; 21.66]$

$2.19[1.03 ; 4.66]$

$0.58[0.07 ; 4.98]$

$0.53[0.21 ; 1.34]$

$3.64[0.69 ; 19.19]$

$3.43[0.48 ; 24.70]$

$2.57[0.26 ; 25.07]$

$3.00[0.35 ; 25.46]$

$2.57[0.26 ; 25.07]$

$2.57[0.65 ; 10.21]$

$0.52[0.07 ; 3.63]$

$3.00[0.35 ; 25.46]$

$0.61 \quad[0.14 ; 2.63]$

$1.98[0.53 ; 7.46]$

$0.81 \quad[0.20 ; 3.28]$

$2.30[0.39 ; 13.47]$

$0.12[0.01 ; 1.37]$

$1.00 \quad[0.15 ; 6.47]$

$3.00[0.35 ; 25.46]$

$2.39 \quad[0.84 ; 6.79]$

$1.80[0.18 ; 17.64]$

$3.43[0.32 ; 36.26]$

$1.98[0.28 ; 14.21]$

$0.37 \quad[0.03 ; 4.07]$

$4.66[0.61 ; 35.69]$

$0.16[0.01 ; 1.71]$

$0.58 \quad[0.07 ; 4.98]$

$3.21[0.33 ; 30.80]$

$1.00 \quad[0.21 ; 4.80]$

$1.80[0.18 ; 17.64]$

$5.17[1.05 ; 25.46]$

$4.74[0.64 ; 35.20]$

$0.59 \quad[0.10 ; 3.41]$

$1.27[0.40 ; 4.00]$

$1.61[0.25 ; 10.21]$

$0.04[0.00 ; 0.76]$

$3.83[0.68 ; 21.66]$

$3.45[0.56 ; 21.10]$

$2.68 \quad[0.75 ; 9.54]$

$1.00 \quad[0.12 ; 8.34]$

$1.38 \quad[0.54 ; 3.54]$

$1.74[0.12 ; 25.46]$

$6.34[0.59 ; 68.62]$

$3.74[0.54 ; 25.86]$

$1.00 \quad[0.11 ; 8.84]$

$0.43 \quad[0.09 ; 2.17]$

$1.69[0.22 ; 13.04]$

$0.31 \quad[0.03 ; 3.00]$

$1.47[1.17 ; 1.85]$

$1.47[1.17 ; 1.85]$
$1.0 \%$

$1.8 \%$

$3.5 \%$

$1.4 \%$

$1.7 \%$

$9.1 \%$

$1.1 \%$

$6.0 \%$

$1.9 \%$

$1.3 \%$

$1.0 \%$

$1.1 \%$

$1.0 \%$

$2.7 \%$

$1.4 \%$

$1.1 \%$

$2.4 \%$

$3.0 \%$

$2.7 \%$

$1.7 \%$

$0.9 \%$

$1.5 \%$

$1.1 \%$

$4.8 \%$

$1.0 \%$

$0.9 \%$

$1.3 \%$

$0.9 \%$

$1.3 \%$

$0.9 \%$

$1.1 \%$

$1.0 \%$

$2.1 \%$

$1.0 \%$

$2.0 \%$

$1.3 \%$

$1.7 \%$

$3.9 \%$

$1.5 \%$

$0.6 \%$

$1.7 \%$

$1.6 \%$

$3.2 \%$

$1.2 \%$

$5.8 \%$

$0.7 \%$

$0.9 \%$

$1.4 \%$

$1.1 \%$

$2.0 \%$

$1.2 \%$

$1.0 \%$

$100 \%$
$1.0 \%$

$1.8 \%$

$3.5 \%$

$1.4 \%$

$1.7 \%$

$9.1 \%$

$1.1 \%$

$6.0 \%$

$1.9 \%$

$1.3 \%$

$1.0 \%$

$1.1 \%$

$1.0 \%$

$2.7 \%$

$1.4 \%$

$1.1 \%$

$2.4 \%$

$3.0 \%$

$2.7 \%$

$1.7 \%$

$0.9 \%$

$1.5 \%$

$1.1 \%$

$4.8 \%$

$1.0 \%$

$0.9 \%$

$1.3 \%$

$0.9 \%$

$1.3 \%$

$0.9 \%$

$1.1 \%$

$1.0 \%$

$2.1 \%$

$1.0 \%$

$2.0 \%$

$1.3 \%$

$1.7 \%$

$3.9 \%$

$1.5 \%$

$0.6 \%$

$1.7 \%$

$1.6 \%$

$3.2 \%$

$1.2 \%$

$5.8 \%$

$0.7 \%$

$0.9 \%$

$1.4 \%$

$1.1 \%$

$2.0 \%$

$1.2 \%$

$1.0 \%$

$--\quad 100 \%$

FIGURE 3 | Forest plot of Wald ratios and 95\% Cls from BMI-associated SNPs. 


\section{- MR-Egger test}

To ensure that violations of our analysis were not biasing the estimate of the directional causal association, MR-Egger regression asymmetry test was used (Bowden et al., 2015). The MR-Egger regression is adapted from Egger regression, which is a tool to detect small study bias in meta-analysis and test for bias from pleiotropy. The estimated value of the intercept in MREgger regression can be interpreted as an estimate of the average pleiotropic effect across the genetic variants. An intercept that differs from zero is indicative of overall directional pleiotropy. The slope coefficient from MR-Egger regression provides a bias estimate of the causal effect.

All statistical tests for MR analysis were undertaken using the R Package of meta-analysis ${ }^{1}$ and Mendelian Randomization (Yavorska and Burgess, 2017).

\section{RESULTS}

Among the 97 BMI-associated SNPs (Locke et al., 2015), 19 SNPs with LDs, 2 T2DM-associated SNPs (rs7138803, rs10938397) from Xi et al. (2014) study, 20 T2DM-associated SNPs and 1 unmapped SNPs from Morris et al. (2012) study, and 3 uncertain SNPs were removed (Supplementary Table 1). 52 BMI-associated SNPs were eventually selected for the MR analysis in Table 1. Each line of the table documents 12 items involving the SNP, EA and its frequencies, beta coefficients of the SNP on the risk of BMI and T2DM, and SEs.

\section{The Influence of BMI on the Risk of T2DM}

The pooled results using IVW method from 52 individual SNPs showed that high BMI significantly increases the risk of T2DM. Due to the lack of evidence of heterogeneity between variants of the summarized data $\left(P=0.499\right.$ and $I^{2}=0 \%$; Figure 3), the fixed-effect model was utilized here for meta-analysis. The OR of T2DM per $5 \mathrm{~kg} / \mathrm{m}^{2}$ higher BMI was 1.470 (95\% CI 1.170 to 1.847; $P=0.001$ ). In addition, we analyzed the effect of BMI on the risk of T2DM by six other methods involving Simple median, Weighted median, Penalized weighted median, Penalized IVW, Robust IVW, and Penalized robust IVW methods (Zhao et al., 2017). The results were shown in Table 2, which are consistent with the result based on IVW method.

\section{Sensitivity Analysis}

ORs from leave-one-out analysis were shown in Figure 4. In comparison with the observed result (1.470) from 52 SNPs, the OR increased by 0.075 [(1.568 - 1.470) / 1.470] after removing rs 10182181. The ORs after removing other SNPs range from 1.412 to 1.507 , which means that the small fluctuation ffrom $-0.039[(1.412-1.470) / 1.470]$ to $0.025[(1.507-1.470)$ / 1.470]\} can be activated by most of the individual SNPs. These results demonstrated that no single SNP drives the IVW point estimate. The detailed results about Heterogeneity test and

\footnotetext{
${ }^{1}$ http://cran.r-project.org/web/packages/meta/index.html
}

TABLE 2 | Associations of genetic variants with BMl and T2DM.

\begin{tabular}{lcccc}
\hline Method & OR & Lower OR & Upper OR & P-value \\
\hline Simple median & 1.767 & 1.252 & 2.492 & 0.001 \\
Weighted median & 1.790 & 1.270 & 2.524 & 0.001 \\
Penalized weighted median & 1.956 & 1.383 & 2.770 & 0.000 \\
Penalized IVW & 1.531 & 1.215 & 1.931 & 0.000 \\
Robust IWW & 1.542 & 1.178 & 2.016 & 0.003 \\
Penalized robust IWW & 1.573 & 1.240 & 1.998 & 0.000 \\
\hline
\end{tabular}

meta-analysis of the leave-one-out analysis were shown in the Supplementary Table 2.

\section{Pleiotropic Effect Analysis}

Figure 5 shows the symmetrical inverted funnel of the point estimate from individual variants. The effect estimated from MREgger regression was 1.24 (95\% CI 0.553 to $1.928 ; P=0.493$ ), with an intercept of 0.004 (95\% CI -0.013 to $0.020 ; P=0.661$; Figure 6). Together these findings provided evidence against the possibility that horizontal pleiotropic effects tend to be bias IVW estimates.

\section{DISCUSSION}

In this study, we exposed the causal effect of BMI on the risk of T2DM using MR method. Here, two-summary level data involving association between genetic variants and BMI from Locke et al. (2015) study and association between genetic variants and T2DM from Morris et al. (2012) study were utilized for this purpose. According to the previous investigation, the MR was viewed as a meta-analysis of multiple genetic variants (Bowden et al., 2015; Nordestgaard et al., 2017; Noyce et al., 2017; Wei et al., 2017). Since there was very low heterogeneity between variants of the summarized data $\left(P=0.499\right.$ and $\left.I^{2}=0 \%\right)$ (Figure 3), the fixed-effect model was utilized for meta-analysis. The pooled results of point estimates using IVW method indicate that the OR of T2DM per $5 \mathrm{~kg} / \mathrm{m}^{2}$ higher BMI was 1.470 (95\% CI 1.170 to $1.847 ; P=0.001$ ). This evidence suggested that high BMI increases the risk of T2DM.

Sensitivity analysis and bias analysis were then carried out for genetic variants. To test whether the results are influenced by individual SNPs, we conducted the leave-one-out validation. Results in Figure 4 indicate very small fluctuations after the removal of individual SNPs. The statistical evidence of MR-Egger regression $(P=0.493)$ with a very low intercept $(0.004$; Figure 6) indicates no significant bias of our data and no pleiotropic effect of the genetic variants, respectively.

The inference that the causal effect of BMI on the risk of T2DM from this study is valuable for both investigations and clinical practice. Although abundant observational studies identified the association between BMI and T2DM, a causal effect cannot be ascertained from these investigations. Especially when their common SNPs were identified in recent studies, these genetic variants were then deemed as the primary cause of the BMI-T2DM association by some of the researchers. In brief, current studies cannot help to understand how BMI is associated 


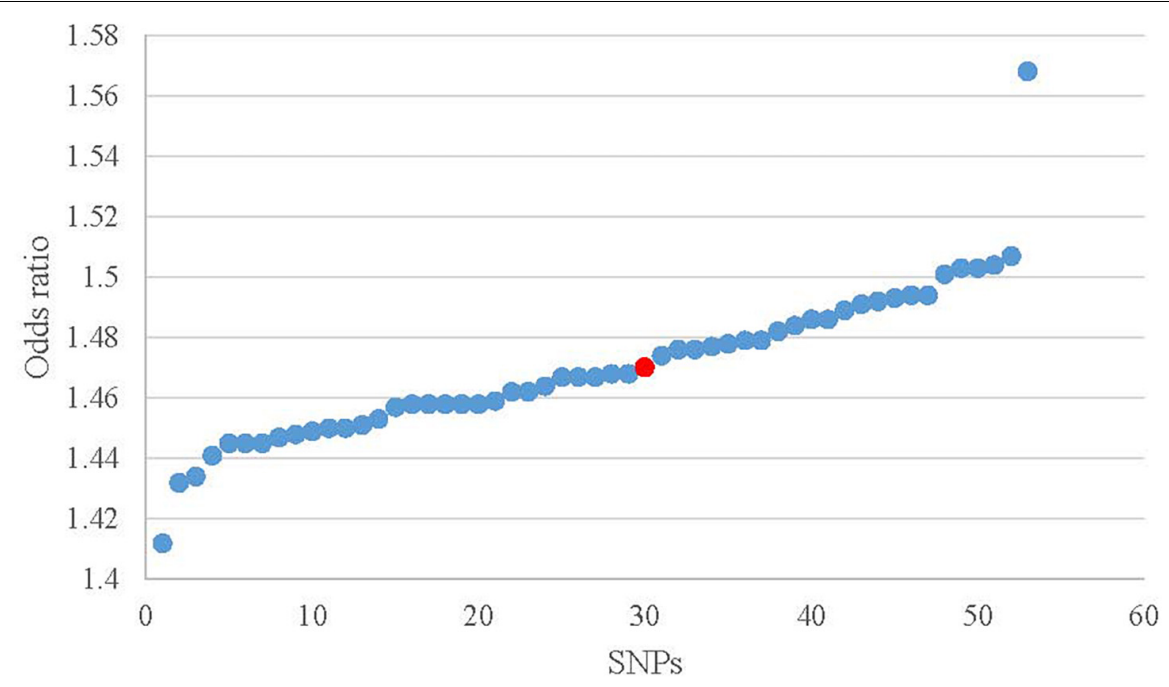

FIGURE 4 | Scatter plot of the ORs in leave-one-out analysis. Red dot is the result without missing SNPs. Blue dots represent the results after missing one SNP.

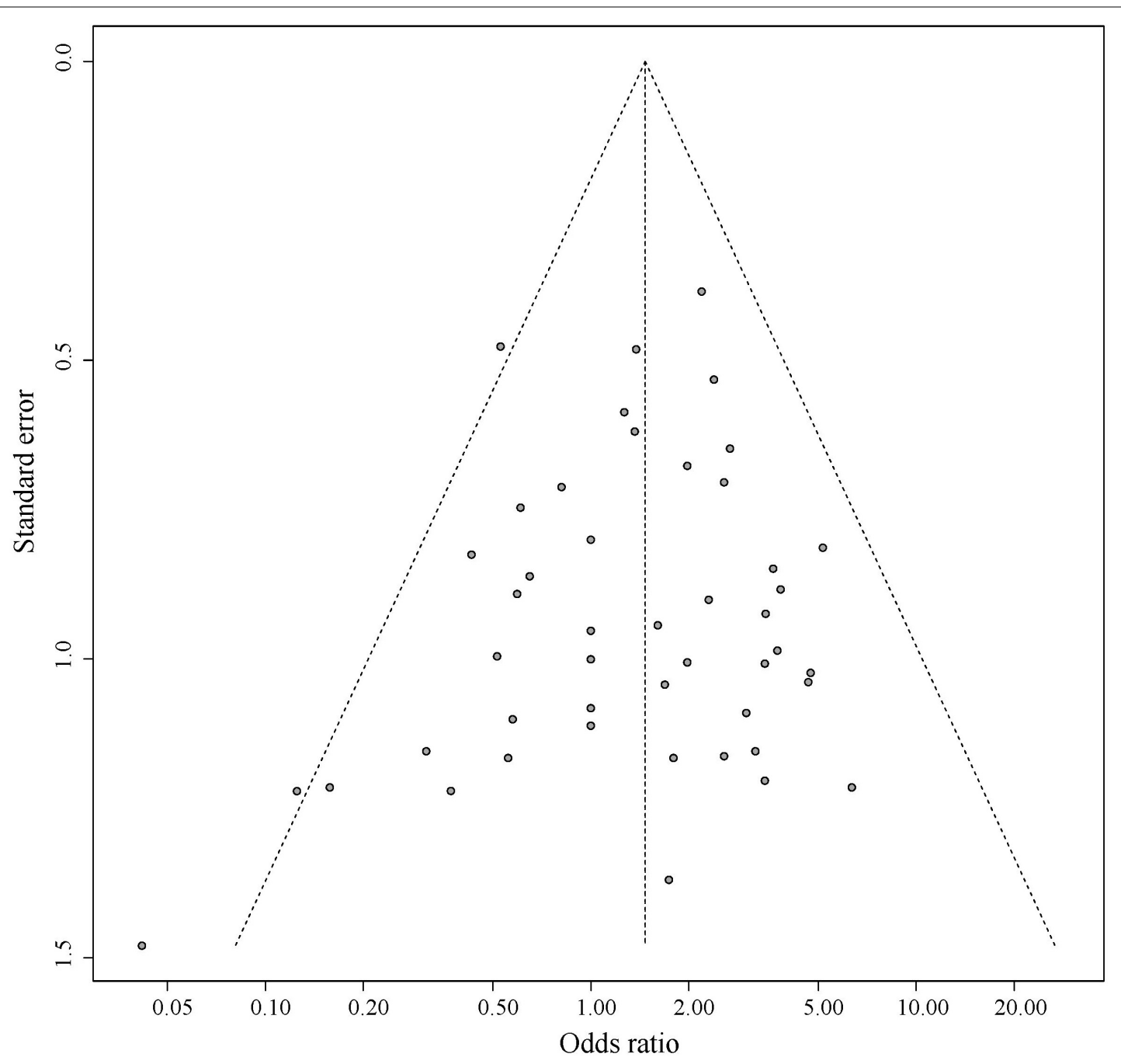

FIGURE 5 | Funnel plot for pleiotropic effect analysis of the variants. 


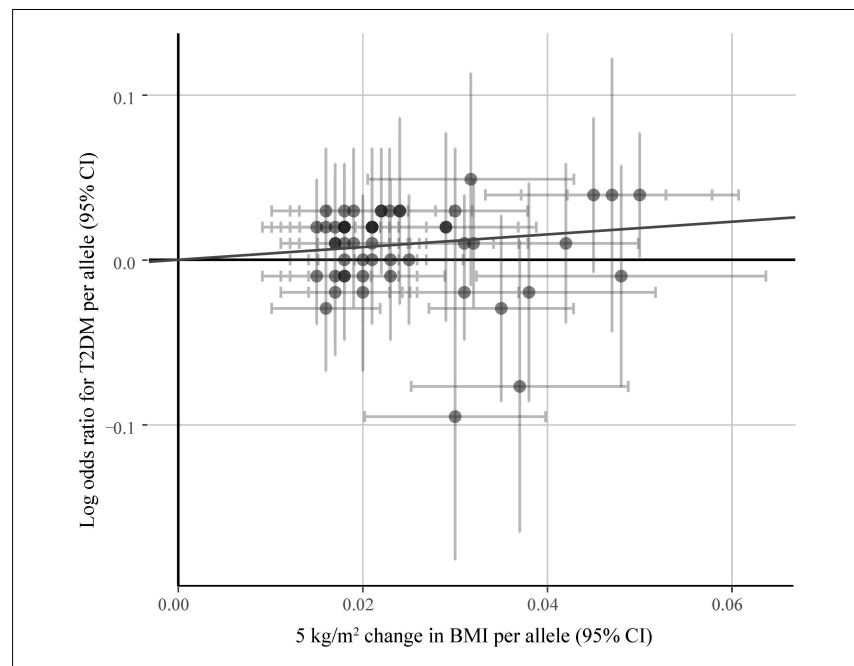

FIGURE 6 | The associations of individual SNPs with BMI and T2DM. Bars represent $95 \% \mathrm{Cls}$. The slopes of the blue and black lines show the estimates of genetic variants using IVW method and MR-Egger method, respectively.

with T2DM. The observation of this causal effect suggested that helping to decline BMI could be used as a potential method when developing T2DM prevention strategies. Excessive BMI means that the body is overweight or in most cases obese, and this is most likely as the real initial cause of T2DM. Obesity has become a pandemic disease worldwide, which has resulted in a significant increase in the incidence of diabetes, non-alcoholic fatty liver disease and coronary heart disease (Milic et al., 2014; Rao et al., 2015; Zhou et al., 2017). In obesity, the hypertrophy, hypoxia of fat cells, endoplasmic reticulum stress, lipids toxicity and many other factors can lead to adipocytokines dysfunction, increased vascular permeability, along with promoting immune cell infiltration into fat tissue, release of more inflammatory factors, and formation of a vicious circle of inflammatory reactions, leading to the persistence of chronic inflammatory states. It is now widely believed that inflammation plays a key mediator role in the development of type 2 diabetes (Ramalho and Guimaraes, 2008; Engin, 2017). Therefore, strengthening exercise, maintaining a reasonable diet and good fitness are still the iron we must adhere to.

Our study benefits from both the GWAS data and MR method. Clinical statistics using typical methods exposed large number of the associations between diseases and phenotypic exposures. With the rapid increase in the identifications about the genetic basis of diseases and phenotypic exposures, using genetic variants for precise estimates of the causal effect of phenotype on disease by MR method, attracts more and more attention (Benn et al., 2017; Richmond et al., 2017; Rodriguez-Broadbent et al., 2017; Went et al., 2017). For example, Noyce et al. (2017) utilized the MR method for assessing the causal influence of BMI on the risk of Parkinson disease (PD). Nordestgaard et al. (2017) estimated the effect of BMI on Alzheimer's disease (AD). On account of multiple genetic variants of phenotypes, Bowden et al. (2015) proposed a strategy to view MR with multiple instruments as a meta-analysis and an MR-Egger method for analyzing bias caused by pleiotropy, which was widely used in current studies. Considering the fuzzy relation between BMI and T2DM, we conducted this MR analysis to specify their relationship.

The two assumptions were described in the "Introduction" section for our MR study. Following assumption 1, 97 BMIassociated SNPs were extracted from summary-level data of Locke et al. (2015) study. After removing SNPs with LD and T2DM-associated SNPs, 52 SNPs conforming to the assumption 2 were assigned for further analysis. In addition, MR requires that the genetic variants are independent of any known confounding variables. During to the lack of information about potential confounding factors of BMI and T2DM, no confounders were considered in this study. Therefore, our observation may be limited by this weakness. Link prediction (Liu et al., 2017; Zhang et al., 2017; Peng et al., 2018a) and artificial intelligence methods (Cabarle et al., 2017; Peng et al., 2018b; Wei et al., 2017, 2018b,c) may be used to solve this problem, which has been successfully applied in the prediction of disease genes (Peng et al., 2017; Zeng et al., 2017), miRNAs (Zeng et al., 2016, 2018; Zou et al., 2016), RNA methylation (Wei et al., 2018a), and drug-induced hepatotoxicity (Su et al., 2018).

In summary, the MR analysis in this article verified that high BMI can increase the risk of T2DM. It helps us to understand the pathogenic factor of T2DM. It also may help to enhance the molecular and phenotypic annotations of T2DM and human diseases (Cheng et al., 2016, 2018c), which could be further applied in analyzing diseases in a system biology perspective (Cheng et al., 2018a,b; Hu et al., 2018).

\section{AUTHOR CONTRIBUTIONS}

LC, JH, RT, and YH conceived and designed the experiments. LC, $\mathrm{HZ}, \mathrm{HJ}$, and SY analyzed the data. LC wrote this manuscript. All authors read and approved the final manuscript.

\section{FUNDING}

This work was supported by the Major State Research Development Program of China (No. 2016YFC1202302), the National Natural Science Foundation of China (Grant No. 61871160, and 61502125), the Heilongjiang Postdoctoral Fund (Grant No. LBH-TZ20, and LBH-Z15179), and the China Postdoctoral Science Foundation (Grant No. 2018T110315, and 2016M590291).

\section{ACKNOWLEDGMENTS}

The authors thank for Guiyou Liu for the improvement of this manuscript.

\section{SUPPLEMENTARY MATERIAL}

The Supplementary Material for this article can be found online at: https://www.frontiersin.org/articles/10.3389/fgene.2019. 00094/full\#supplementary-material 


\section{REFERENCES}

Andreasen, C. H., Stender-Petersen, K. L., Mogensen, M. S., Torekov, S. S., Wegner, L., Andersen, G., et al. (2008). Low physical activity accentuates the effect of the FTO rs 9939609 polymorphism on body fat accumulation. Diabetes Metab. Res. Rev. 57, 95-101. doi: 10.2337/db07-0910

Benn, M., Nordestgaard, B. G., Frikke-Schmidt, R., and Tybjærg-Hansen, A. (2017). Low LDL cholesterol, PCSK9 and HMGCR genetic variation, and risk of Alzheimer's disease and Parkinson's disease: Mendelian randomisation study. BMJ 357:j3170. doi: 10.1136/bmj.j1648

Bowden, J., Smith, G. D., and Burgess, S. (2015). Mendelian randomization with invalid instruments: effect estimation and bias detection through egger regression. Int. J. Epidemiol. 44, 512-525. doi: 10.1093/ije/dyv080

Cabarle, F. G. C., Adorna, H. N., Jiang, M., and Zeng, X. (2017). Spiking neural P systems with scheduled synapses. IEEE Trans. Nanobiosci. 16, 792-801. doi: 10.1109/TNB.2017.2762580

Cauchi, S., Stutzmann, F., Cavalcanti-Proenca, C., Durand, E., Pouta, A., Hartikainen, A. L., et al. (2009). Combined effects of MC4R and FTO common genetic variants on obesity in european general populations. J. Mol. Med. 87, 537-546. doi: 10.1007/s00109-009-0451-6

Chen, F., Guo, Z., Wu, M., Zhou, Z., and Luo, W. (2015). [Impact of dynamic changes of waist circumference and body mass index on type 2 diabetes mellitus risk]. Zhonghua Yu Fang Yi Xue Za Zhi 49, 1092-1097.

Chen, X. Y., Wu, Z. F., Wang, X. C., Dong, X. L., Zhu, J. F., Chen, T., et al. (2016). [Association between body mass index and its change and type 2 diabetes mellitus risk in a prospective study]. Zhonghua Liu Xing Bing Xue Za Zhi 37, 1332-1335. doi: 10.3760/cma.j.issn.0254-6450.2016.10.003

Cheng, L., Hu, Y., Sun, J., Zhou, M., and Jiang, Q. (2018a). DincRNA: a comprehensive web-based bioinformatics toolkit for exploring disease associations and ncRNA function. Bioinformatics 34, 1953-1956. doi: 10.1093/ bioinformatics/bty002

Cheng, L., Jiang, Y., Ju, H., Sun, J., Peng, J., Zhou, M., et al. (2018b). InfAcrOnt: calculating cross-ontology term similarities using information flow by a random walk. BMC Genomics 19:919. doi: 10.1186/s12864-017-4338-6

Cheng, L., Wang, P., Tian, R., Wang, S., Guo, Q., Luo, M., et al. (2018c). LncRNA2Target v2.0: a comprehensive database for target genes of lncRNAs in human and mouse. Nucleic Acids Res. 47, D140-D144. doi: 10.1093/nar/ gky1051

Cheng, L., Sun, J., Xu, W., Dong, L., Hu, Y., and Zhou, M. (2016). Oahg: an integrated resource for annotating human genes with multi-level ontologies. Sci. Rep. 6:34820. doi: 10.1038/srep34820

Devuyst, O. (2015). The 1000 genomes project: welcome to a new World. Perit. Dial. Int. 35, 676-677. doi: 10.3747/pdi.2015.00261

Engin, A. (2017). The pathogenesis of obesity-associated adipose tissue inflammation. Adv. Exp. Med. Biol. 960, 221-245. doi: 10.1007/978-3-31948382-5_9

Frayling, T. M., Timpson, N. J., Weedon, M. N., Zeggini, E., Freathy, R. M., Lindgren, C. M., et al. (2007). A common variant in the FTO gene is associated with body mass index and predisposes to childhood and adult obesity. Science 316, 889-894. doi: 10.1126/science.1141634

Ganz, M. L., Wintfeld, N., Li, Q., Alas, V., Langer, J., and Hammer, M. (2014). The association of body mass index with the risk of type 2 diabetes: a casecontrol study nested in an electronic health records system in the United States. Diabetol. Metab. Syndr. 6:50. doi: 10.1186/1758-5996-6-50

Herder, C., Rathmann, W., Strassburger, K., Finner, H., Grallert, H., Huth, C., et al. (2008). Variants of the PPARG, IGF2BP2, CDKAL1, HHEX, and TCF7L2 genes confer risk of type 2 diabetes independently of BMI in the German KORA studies. Horm. Metab. Res. 40, 722-726. doi: 10.1055/s-2008-1078730

Hu, Y., Zhao, T., Zhang, N., Zang, T., Zhang, J., and Cheng, L. (2018). Identifying diseases-related metabolites using random walk. BMC Bioinformatics 19:116. doi: 10.1186/s12859-018-2098-1

Lee, S. H., Wray, N. R., Goddard, M. E., and Visscher, P. M. (2011). Estimating missing heritability for disease from genome-wide association studies. Am. J. Hum. Genet. 88, 294-305. doi: 10.1016/j.ajhg.2011.02.002

Legry, V., Cottel, D., Ferrieres, J., Arveiler, D., Andrieux, N., Bingham, A., et al. (2009). Effect of an FTO polymorphism on fat mass, obesity, and type 2 diabetes mellitus in the french monica study. Metabolism 58, 971-975. doi: 10.1016/j. metabol.2009.02.019
Liu, Y., Zeng, X., He, Z., and Zou, Q. (2017). Inferring microRNA-disease associations by random walk on a heterogeneous network with multiple data sources. IEEE-ACM Trans. Comput. Biol. Bioinform. 14, 905-915.

Locke, A. E., Kahali, B., Berndt, S. I., Justice, A. E., Pers, T. H., Day, F. R., et al. (2015). Genetic studies of body mass index yield new insights for obesity biology. Nature 518, 197-206. doi: 10.1038/nature14177

Milic, S., Lulic, D., and Stimac, D. (2014). Non-alcoholic fatty liver disease and obesity: biochemical, metabolic and clinical presentations. World J. Gastroenterol. 20, 9330-9337. doi: 10.3748/wjg.v20.i28.9330

Morris, A. P., Voight, B. F., Teslovich, T. M., Ferreira, T., Segre, A. V., Steinthorsdottir, V., et al. (2012). Large-scale association analysis provides insights into the genetic architecture and pathophysiology of type 2 diabetes. Nat. Genet. 44, 981-990. doi: 10.1038/ng.2383

Nordestgaard, L. T., Tybjaerg-Hansen, A., Nordestgaard, B. G., and FrikkeSchmidt, R. (2017). Body mass index and risk of Alzheimer disease: a mendelian randomization study of 399,536 individuals. J. Clin. Endocrinol. Metab. 102, 2310-2320. doi: 10.1210/jc.2017-00195

Noyce, A. J., Kia, D. A., Hemani, G., Nicolas, A., Price, T. R., De PabloFernandez, E., et al. (2017). Estimating the causal influence of body mass index on risk of Parkinson disease: a mendelian randomisation study. PLoS Med. 14:e1002314. doi: 10.1371/journal.pmed.1002314

Olokoba, A. B., Obateru, O. A., and Olokoba, L. B. (2012). Type 2 diabetes mellitus: a review of current trends. Oman Med. J. 27, 269-273. doi: 10.5001/omj.2012.68

Pan, A., Sun, Q., Manson, J. E., Willett, W. C., and Hu, F. B. (2013). Walnut consumption is associated with lower risk of type 2 diabetes in women. J. Nutr. 143, 512-518. doi: 10.3945/jn.112.172171

Peng, J., Hui, W. W., and Shang, X. Q. (2018a). Measuring phenotype-phenotype similarity through the interactome. BMC Bioinformatics 19:114. doi: 10.1186/ s12859-018-2102-9

Peng, J., Zhang, X., Hui, W., Lu, J., Li, Q., Liu, S., et al. (2018b). Improving the measurement of semantic similarity by combining gene ontology and cofunctional network: a random walk based approach. BMC Syst. Biol. 12:18. doi: 10.1186/s12918-018-0539-0

Peng, J. J., Xue, H. S., Shao, Y. K., Shang, X. Q., Wang, Y. D., and Chen, J. (2017). A novel method to measure the semantic similarity of HPO terms. Int. J. Data Min. Bioinform. 17, 173-188. doi: 10.1504/IJDMB.2017.084268

Ramalho, R., and Guimaraes, C. (2008). [The role of adipose tissue and macrophages in chronic inflammation associated with obesity: clinical implications]. Acta Med. Port. 21, 489-496.

Rao, W. S., Shan, C. X., Zhang, W., Jiang, D. Z., and Qiu, M. (2015). A meta-analysis of short-term outcomes of patients with type 2 diabetes mellitus and BMI $<1$ $=35 \mathrm{~kg} / \mathrm{m} 2$ undergoing Roux-en-Y gastric bypass. World J. Surg. 39, 223-230. doi: 10.1007/s00268-014-2751-4

Richmond, R. C., Timpson, N. J., Felix, J. F., Palmer, T., Gaillard, R., McMahon, G., et al. (2017). Using genetic variation to explore the causal effect of maternal pregnancy adiposity on future offspring adiposity: a mendelian randomisation study. PLoS Med. 14:e1002221. doi: 10.1371/journal.pmed.1002221

Rodriguez-Broadbent, H., Law, P. J., Sud, A., Palin, K., Tuupanen, S., Gylfe, A., et al. (2017). Mendelian randomisation implicates hyperlipidaemia as a risk factor for colorectal cancer. Int. J. Cancer 140, 2701-2708. doi: 10.1002/ijc.30709

Sanada, H., Yokokawa, H., Yoneda, M., Yatabe, J., Sasaki Yatabe, M., Williams, S. M., et al. (2012). High body mass index is an important risk factor for the development of type 2 diabetes. Intern. Med. 51, 1821-1826. doi: 10.2169/ internalmedicine. 51.7410

Shi, Y., and Hu, F. B. (2014). The global implications of diabetes and cancer. Lancet 383, 1947-1948. doi: 10.1016/S0140-6736(14)60886-2

Song, Y., You, N. C., Hsu, Y. H., Howard, B. V., Langer, R. D., Manson, J. A. E., et al. (2012). FTO polymorphisms are associated with obesity but not diabetes risk in postmenopausal women. Obesity 16, 2472-2480. doi: 10.1038/oby. 2008.408

Su, R., Wu, H., Xu, B., Liu, X., and Wei, L. (2018). Developing a multi-dose computational model for drug-induced hepatotoxicity prediction based on toxicogenomics data. IEEE/ACM Trans. Comput. Biol. Bioinform. doi: 10.1109/ TCBB.2018.2858756

Voight, B. F., Kang, H. M., Ding, J., Palmer, C. D., Sidore, C., Chines, P. S., et al. (2012). The metabochip, a custom genotyping array for genetic studies of metabolic, cardiovascular, and anthropometric traits. PLoS Genet. 8:e1002793. doi: 10.1371/journal.pgen.1002793 
Webster, R. J., Warrington, N. M., Beilby, J. P., Frayling, T. M., and Palmer, L. J. (2010). The longitudinal association of common susceptibility variants for type 2 diabetes and obesity with fasting glucose level and BMI. BMC Med. Genet. 11:140. doi: 10.1186/1471-2350-11-140

Wei, L., Chen, H., and Su, R. (2018a). M6APred-EL: a sequence-based predictor for identifying N6-methyladenosine sites using ensemble learning. Mol. Ther. Nucleic Acids 12, 635-644. doi: 10.1016/j.omtn.2018.07.004

Wei, L., Ding, Y., Su, R., Tang, J., and Zou, Q. (2018b). Prediction of human protein subcellular localization using deep learning. J. Parallel Distrib. Comput. 117, 212-217. doi: 10.2174/1566523218666180913110949

Wei, L., Zhou, C., Chen, H., Song, J., and Su, R. (2018c). ACPred-FL: a sequencebased predictor based on effective feature representation to improve the prediction of anti-cancer peptides. Bioinformatics 34, 4007-4016. doi: 10.1093/ bioinformatics/bty 451

Wei, L., Xing, P., Shi, G., Ji, Z., and Zou, Q. (2017). Fast prediction of methylation sites using sequence-based feature selection technique. IEEE/ACM Trans. Comput. Biol. Bioinform. doi: 10.1109/TCBB.2017.2670558 [Epub ahead of print].

Went, M., Sud, A., Law, P. J., Johnson, D. C., Weinhold, N., Forsti, A., et al. (2017). Assessing the effect of obesity-related traits on multiple myeloma using a Mendelian randomisation approach. Blood Cancer J. 7:e573. doi: 10.1038/bcj. 2017.48

Xi, B., Takeuchi, F., Meirhaeghe, A., Kato, N., Chambers, J. C., Morris, A. P., et al. (2014). Associations of genetic variants in/near body mass index-associated genes with type 2 diabetes: a systematic meta-analysis. Clin. Endocrinol. 81, 702-710. doi: 10.1111/cen.12428

Yang, J., Benyamin, B., McEvoy, B. P., Gordon, S., Henders, A. K., Nyholt, D. R., et al. (2010). Common SNPs explain a large proportion of the heritability for human height. Nat. Genet. 42, 565-569. doi: 10.1038/ng.608

Yavorska, O. O., and Burgess, S. (2017). Mendelian randomization: an R package for performing mendelian randomization analyses using summarized data. Int. J. Epidemiol. 46, 1734-1739. doi: 10.1093/ije/dyx034

Zeng, X., Liao, Y., Liu, Y., and Zou, Q. (2017). Prediction and validation of disease genes using hetesim scores. IEEE-ACM Trans. Comput. Biol. Bioinform. 14, 687-695. doi: 10.1109/TCBB.2016.2520947
Zeng, X., Zhang, X., and Zou, Q. (2016). Integrative approaches for predicting microRNA function and prioritizing disease-related microRNA using biological interaction networks. Brief. Bioinform. 17, 193-203. doi: 10.1093/bib/ bbv033

Zeng, X. X., Liu, L., Lu, L. Y., and Zou, Q. (2018). Prediction of potential diseaseassociated microRNAs using structural perturbation method. Bioinformatics 34, 2425-2432. doi: 10.1093/bioinformatics/bty112

Zhang, S., Zhang, D., Jiang, Y., Wu, L., Shang, H., Liu, J., et al. (2015). CLU rs2279590 polymorphism contributes to Alzheimer's disease susceptibility in Caucasian and Asian populations. J. Neural Transm. 122, 433-439. doi: 10.1007/ s00702-014-1260-9

Zhang, X., Zou, Q., Rodruguez-Paton, A., and Zeng, X. (2017). Metapath methods for prioritizing candidate disease miRNAs. IEEE/ACM Trans. Comput. Biol. Bioinform. 16, 283-291. doi: 10.1109/TCBB.2017. 2776280

Zhao, Q., Laukkanen, J. A., Li, Q., and Li, G. (2017). Body mass index is associated with type 2 diabetes mellitus in chinese elderly. Clin. Interv. Aging 12, 745-752. doi: 10.2147/CIA.S130014

Zhou, Y., Zhang, Y., Shi, K., and Wang, C. (2017). Body mass index and risk of diabetic retinopathy: a meta-analysis and systematic review. Medicine 96:e6754. doi: 10.1097/MD.0000000000006754

Zou, Q., Li, J., Song, L., Zeng, X., and Wang, G. (2016). Similarity computation strategies in the microRNA-disease network: a survey. Brief. Funct. Genomics 15, 55-64. doi: 10.1093/bfgp/elv024

Conflict of Interest Statement: The authors declare that the research was conducted in the absence of any commercial or financial relationships that could be construed as a potential conflict of interest.

Copyright (c) 2019 Cheng, Zhuang, Ju, Yang, Han, Tan and Hu. This is an open-access article distributed under the terms of the Creative Commons Attribution License (CC BY). The use, distribution or reproduction in other forums is permitted, provided the original author(s) and the copyright owner(s) are credited and that the original publication in this journal is cited, in accordance with accepted academic practice. No use, distribution or reproduction is permitted which does not comply with these terms. 